\title{
Spatial-temporal epidemiology of human Salmonella Enteritidis infections with major phage types (PTs 1, 4, 5b, 8, 13, and 13a) in Ontario, Canada, 2008-2009
}

\author{
Csaba Varga ${ }^{1,2^{*}}$, David L. Pearl ${ }^{1}$, Scott A. McEwen ${ }^{1}$, Jan M. Sargeant ${ }^{1,3}$, Frank Pollari ${ }^{4}$ and Michele T. Guerin ${ }^{1}$
}

\begin{abstract}
Background: In Ontario and Canada, the incidence of human Salmonella enterica serotype Enteritidis (S. Enteritidis) infections have increased steadily during the last decade. Our study evaluated the spatial and temporal epidemiology of the major phage types (PTs) of S. Enteritidis infections to aid public health practitioners design effective prevention and control programs.

Methods: Data on S. Enteritidis infections between January 1, 2008 and December 31, 2009 were obtained from Ontario's disease surveillance system. Salmonella Enteritidis infections with major phage types were classified by their annual health region-level incidence rates (IRs), monthly IRs, clinical symptoms, and exposure settings. A scan statistic was employed to detect retrospective phage type-specific spatial, temporal, and space-time clusters of $S$. Enteritidis infections. Space-time cluster cases' exposure settings were evaluated to identify common exposures.

Results: 1,336 cases were available for analysis. The six most frequently reported $S$. Enteritidis PTs were $8(n=398)$, 13a $(n=218), 13(n=198), 1 \quad(n=132), 5 b(n=83)$, and $4(n=76)$. Reported rates of S. Enteritidis infections with major phage types varied by health region and month. International travel and unknown exposure settings were the most frequently reported settings for PT 5b, 4, and 1 cases, whereas unknown exposure setting, private home, food premise, and international travel were the most frequently reported settings for PT 8, 13, and 13a cases.

Diarrhea, abdominal pain, and fever were the most commonly reported clinical symptoms. A number of phage typespecific spatial, temporal, and space-time clusters were identified. Space-time clusters of PTs 1, 4, and 5b occurred mainly during the winter and spring months in the North West, North East, Eastern, Central East, and Central West regions. Space-time clusters of PTs 13 and 13a occurred at different times of the year in the Toronto region. Space-time clusters of PT 8 occurred at different times of the year in the North West and South West regions.

Conclusions: Phage type-specific differences in exposure settings, and spatial-temporal clustering of $S$. Enteritidis infections were demonstrated that might guide public health surveillance of disease outbreaks. Our study methodology could be applied to other foodborne disease surveillance data to detect retrospective high disease rate clusters, which could aid public health authorities in developing effective prevention and control programs.
\end{abstract}

Keywords: Phage type, Surveillance, Retrospective, Travel, Restaurant, Scan statistic, Salmonella Enteritidis, Canada

\footnotetext{
* Correspondence: cvarga@uoguelph.ca

${ }^{1}$ Department of Population Medicine, Ontario Veterinary College, University

of Guelph, Guelph, ON N1G 2W1, Canada

${ }^{2}$ Ontario Ministry of Agriculture, Food and Rural Affairs, Guelph, ON N1G 4Y2,

Canada

Full list of author information is available at the end of the article
} 


\section{Background}

Salmonellosis is a major foodborne bacterial infection that continuously poses a significant human health burden worldwide [1]. In Canada, salmonellosis is the main cause of hospitalization and death among domestically acquired foodborne infections [2], causing an estimated 87,510 illnesses annually [3]. In the last decade, Salmonella enterica serotype Enteritidis ( $\boldsymbol{S}$. Enteritidis) became the top serovar among the non-typhoidal salmonellae in Canada [4], the United States of America (US) $[5,6]$, and the European Union [7].

Currently in Canada, the predominant $S$. Enteritidis phage types (PTs) among human cases are PT 8, 13a, 13, 1, 4, and 5b [4]. Between 2006 and 2010, Canadian integrated surveillance systems identified the emergence of PT 13a and an increase in the number of cases of PT 8 [4].

Several research studies conducted in North America have evaluated phage type-specific risk factors for $S$. Enteritidis infections in humans. In Ontario, Canada, researchers demonstrated that cases with PT 8 were more likely to have had contact with dogs compared to cases with other phage types [8]. In British Columbia, Canada, a concurrent increase in the incidence of $S$. Enteritidis infections with PT 8 in humans and the prevalence of PT 8 in poultry was observed between 2007 and 2010 [9]. The researchers demonstrated increased odds of infection with PT 8 in human cases who consumed illegally-sourced ungraded eggs compared to controls [9]. In Alberta, Canada, an outbreak of PT 8, 13, and atypical PTs was linked to the consumption of food products purchased from mobile lunch trucks that were contaminated by illegally-obtained eggs and/or by infected food handlers [10]. In the US, PT 8 cases were more likely to have consumed chicken or be the owner of a lizard than controls, whereas PT 13 cases were more likely to have eaten undercooked eggs in their home than controls [11].

In Ontario and Canada, an increase in the reported number of human $S$. Enteritidis cases was observed during the last decade $[12,13]$. Current Ontario studies revealed that the majority of $S$. Enteritidis cases with PT 1, 4 , or 6 a were international travel-related, whereas cases with PT 8,13 , or 13 a were mainly acquired domestically $[14,15]$. These studies provided valuable information on the seasonality and exposure locations of $S$. Enteritidis cases, although they lacked information on cases' geographical distribution and spatial-temporal clustering. Identifying areas with high rates of reported $S$. Enteritidis cases can be useful for targeting prevention and control programs $[12,16]$.

There have been a limited number of studies that evaluated foodborne disease surveillance data by incorporating geographical information system (GIS) data, spatial- temporal scan statistic results, exposure setting information, and clinical syndrome history. Scan statistics have been effectively used to evaluate clustering and transmission dynamics of pandemic influenza A (H1N1) in Hong Kong, China [17], to detect Escherichia coli O157:H7 outbreaks involving common molecular subtypes in Alberta, Canada [18], to identify the location of high and low rate areas of campylobacteriosis incidence in Manitoba, Canada [19], to identify high incidence clusters of tuberculosis in Linyi City, China [20], and to find childhood cancer clusters in Alberta, Canada [21].

This study assesses the spatial and temporal epidemiology of the phage types of $S$. Enteritidis that predominate in Ontario health regions by: 1) estimating phage type-specific health region-level incidence rates (IRs); 2) estimating phage type-specific monthly IRs; 3) describing phage type-specific exposure settings and clinical symptoms; 4) detecting phage-type-specific spatial, temporal, and space-time clusters of cases; and 5) examining the exposure settings of cases identified within space-time clusters. The results of this study are expected to assist public health officials with the development of disease prevention programs within the province.

\section{Methods}

\section{Study setting and data sources}

Our study was conducted in Ontario, Canada. In 2009, an estimated 13 million people lived in Ontario, accounting for $39 \%$ of Canada's total population [22]. There are 36 public health units (PHUs) in Ontario that are mandated by the provincial ministry of health to administer health promotion and disease prevention programs [23]. These PHUs are grouped into seven planning regions, which were used for the purposes of our study (Fig. 1; Additional file 1: Legend 1).

Salmonellosis is a reportable disease under provincial legislation [23], and is diagnosed by public health, hospital, and private laboratories after isolation of Salmonella spp. (excluding Salmonella Typhi or Paratyphi) from stool (the majority of samples), rectal swabs, urine, blood, or any other sterile site [24]. All Salmonella isolates are sent to the Public Health Ontario Laboratories-Toronto for confirmation and serotyping using serological confirmation of compatible somatic and flagellar antigens (Kauffmann-White classification) [25]. All isolates serotyped as $S$. Enteritidis are sent to the National Microbiology Laboratory in Winnipeg, Manitoba for phage typing using techniques defined by Ward and colleagues [26].

Staff at each PHU in Ontario must follow up with every $S$. Enteritidis case to identify exposure settings during the illness incubation period and the clinical symptoms during illness. Case investigation records must be reported to the Ontario Ministry of Health 


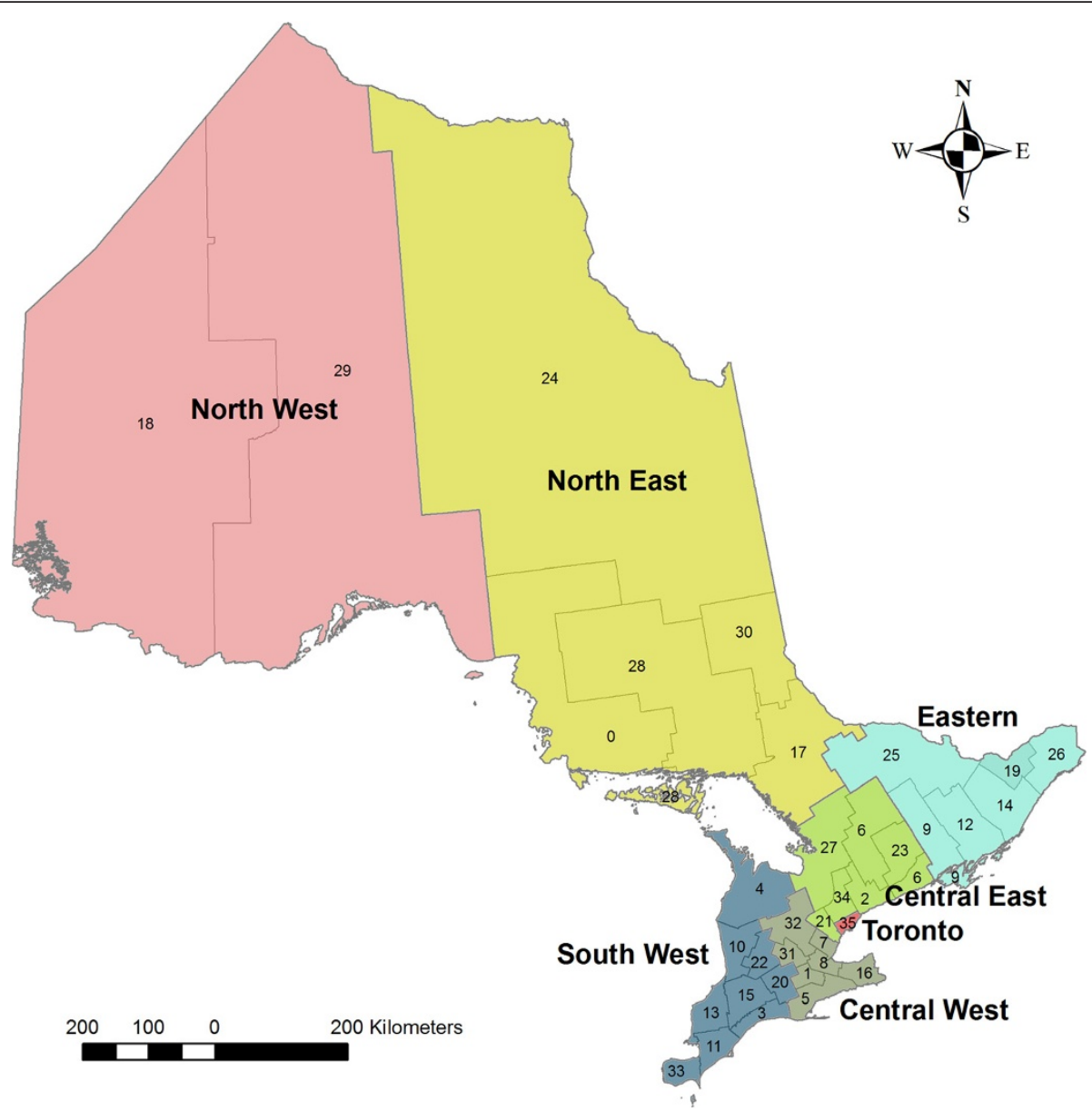

Fig. 1 Health regions in Ontario, Canada. The names and population estimates for the public health units (indicated by labels 0 through 35), are presented in Additional file 1: Legend 1

and Long-Term Care (MOHLTC) through the integrated Public Health Information System (iPHIS). Each PHU has its own case follow-up protocol, and currently there is no standardized follow-up form or set timeline for initial case contact. The exposure setting information is based on what the case reported and was considered significant by the investigator. Exposure settings in the surveillance database were categorized as: international travel (i.e., travelled outside of Canada), private home, food premises (e.g., restaurant, grocery store, bakery, deli, caterer, mobile food premise), other (e.g., institution, hospital, farm, petting zoo, child care centre), or unknown (if the only exposure reported was "unknown"). Cases without exposure setting details were excluded from the exposure setting analysis. When more than one exposure setting was reported, the primary exposure was included in our analysis. Secondary exposure was only considered when the primary exposure was reported as "unknown".

\section{Statistical analysis}

Data management

Data pertaining to the $S$. Enteritidis cases' phage type, age, sex, reporting PHU, date of illness onset, exposure setting, and clinical symptoms were acquired from the iPHIS passive surveillance database. Data were entered into a spreadsheet program (Microsoft Excel 2010, Microsoft Corporation, Redmond, WA, US), reviewed for missing values, and subsequently imported into STATA Intercooled statistical software, version 10.1 (Stata Corporation, College Station, TX, US) for descriptive statistical analysis. Data were available from January 1, 2007 to December 31, 2009; however, due to the large amount of missing phage type information in 2007, all cases from 2007 were excluded from the analyses. Therefore, we evaluated all $S$. Enteritidis cases that were captured within the iPHIS database between January 1, 2008 and December 31, 2009. The frequency of Salmonella Enteritidis phage types was calculated, the most commonly reported phage types were identified ( $>5 \%$ of 
the total number of $S$. Enteritidis cases that were phage typed during the 2-year study period), and the spatial and temporal epidemiology of these phage types were assessed by following several analytical steps, which are outlined in Fig. 2 and described in detail below. Cartographical boundary files and population estimates for each health region were acquired from Statistics Canada [27].

\section{Phage type-specific incidence rates}

Health region-level IRs for the six most commonly reported $S$. Enteritidis phage type cases were calculated by dividing the number of cases in a health region with the phage type during the 2-year study period by the population estimate for the health region for the 2-year study period. Health region-level phage-type specific IRs were illustrated in choropleth maps using ArcGIS 10 software (ESRI Inc., Redlands, CA, US).

For the entire province, monthly IRs for the six most frequent phage types were calculated by dividing the number of cases in a month with the phage type by the monthly population estimate. Smoothed IRs based on a simple 3-month moving average were calculated in Microsoft Excel 2010 and plotted together with the monthly raw IRs.

\section{Scan statistic}

Individual models were built for the 2-year study period for the six most frequent phage types in Ontario. Scan statistics using discrete Poisson models [28] in SaTScan software version 9.0 [29] were conducted to identify purely spatial, purely temporal, and space-time clusters of $S$. Enteritidis cases. The assumption of the Poisson model is that the number of cases in each health region are Poisson-distributed, based on a known underlying population at risk $[28,30]$. Cartesian coordinates of latitude and longitude for each health region centroid were calculated in ArcGIS 10. The smallest spatial and temporal unit was the centroid of a health region and the month of disease onset, respectively. Only high rate clusters were investigated. Secondary clusters were reported if they did not overlap in space with the primary cluster. The scan statistic uses a circular scanning window in space, an interval in time, and a cylinder with a circular spatial base and height corresponding to time in spacetime $[28,30]$. The scanning window of variable radii gradually moves through time and/or space comparing the rate of cases inside the scanning window to outside the window. When the rate inside the scanning window compared to outside is higher than expected by random chance alone, a high rate cluster is identified. A relative

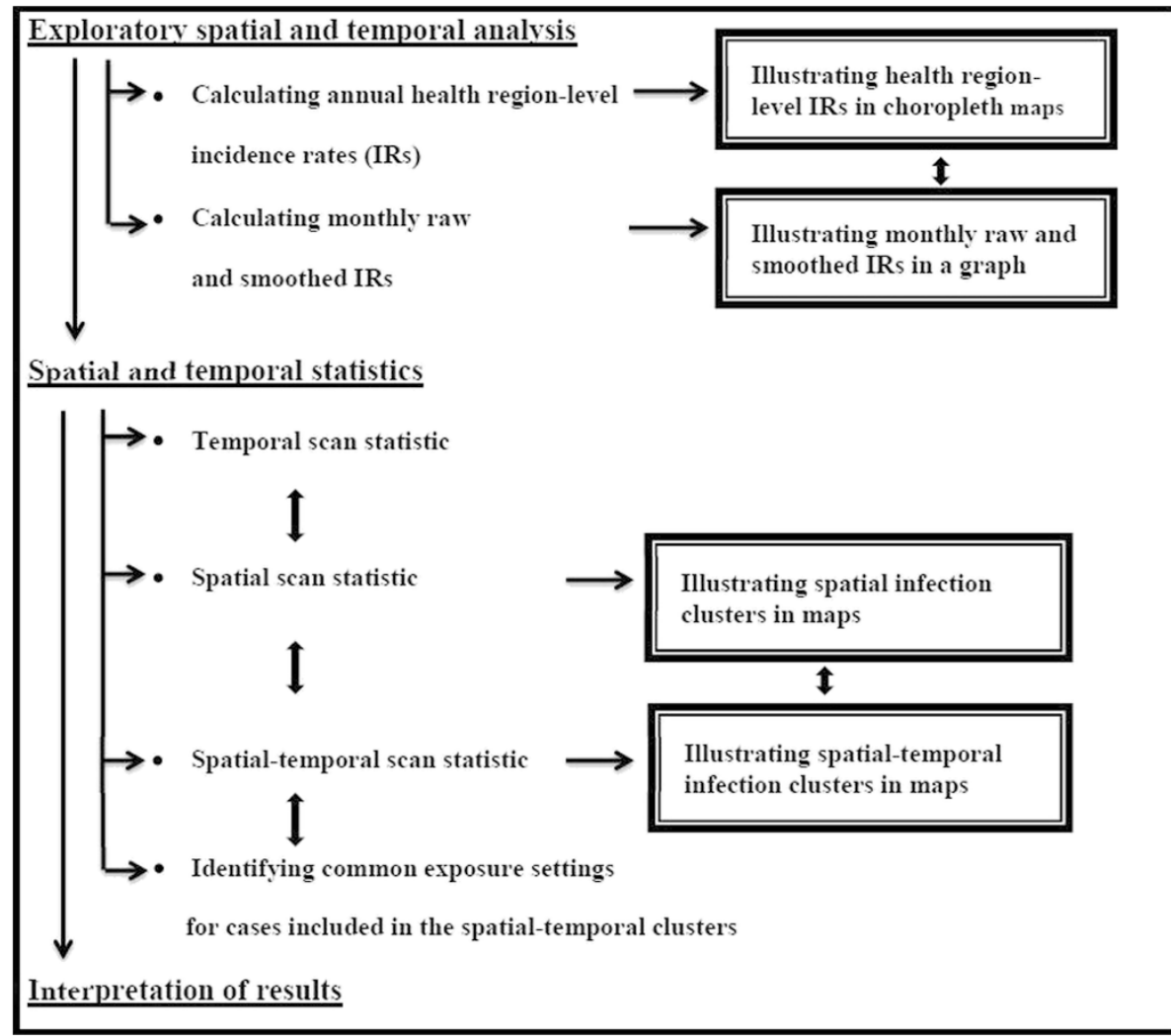

Fig. 2 Flow chart outlining the analytical steps used to evaluate Salmonella Enteritidis cases with major phage types 
risk and a $p$-value obtained through Monte Carlo hypothesis testing using 999 replications were estimated for each cluster [31]. A $p$-value $\leq 0.05$ was considered to be significant. The maximum scanning window size was set to include up to $50 \%$ of the population at risk and up to $50 \%$ of study period $[28,30]$. Analyses were adjusted for age $(0-9,10-24,25-34,35-49, \geq 50$ years) and sex covariates [28]. Statistically significant spatial and space-time clusters were illustrated using a map with health region boundaries in ArcGIS 10. The exposure settings of cases that were part of statistically significant space-time clusters were obtained from iPHIS and examined to assess if a common exposure explained the clustering of cases in space-time.

\section{Results}

A total of $1,364 S$. Enteritidis cases were recorded in the iPHIS database during the study period; of these, 28 cases were missing phage type information, leaving 1,336 cases $(97.9 \%)$ available for analysis. The most commonly reported phage types were PT $8(n=398)$, PT 13a $(n=218)$, PT $13(n=198)$, PT $1(n=132)$, PT $5 \mathrm{~b}(n=83)$, and PT 4 $(n=76)$, which together accounted for $82.7 \%$ of all $S$. Enteritidis cases in Ontario with known phage types during the study period (Table 1). One PT 8 case was excluded from the scan statistics because of missing sex information. No outbreaks (e.g., two or more cases linked epidemiologically) were declared by the MOHLTC during the study period.

\section{Health region-level incidence rates}

Figure 3 illustrates the annual health region-level IRs of S. Enteritidis infections per 100,000 person-years for the six most frequent phage types in Ontario, and described below. For PT 1, the IR ranged from 0.25 to 0.62 units (mean $=0.48$ ), with the highest IRs observed in the Central West and Central East regions. For PT 4, the IR ranged from 0.09 to 0.44 units (mean $=0.25)$, with the

Table 1 Frequency of Salmonella Enteritidis cases with different phage types in Ontario, Canada, 2008-2009 $(n=1,336)$

\begin{tabular}{llll}
\hline Phage type & $\mathrm{n}(\mathrm{n} / \mathrm{N} \%)$ & Phage type & $\mathrm{n}(\mathrm{n} / \mathrm{N} \%)$ \\
\hline PT 8 & $398(29.8)$ & PT 1a & $11(0.8)$ \\
PT 13a & $218(16.3)$ & PT 21 & $11(0.8)$ \\
PT 13 & $198(14.8)$ & PT 1b & $10(0.7)$ \\
PT 1 & $132(9.9)$ & PT 14b & $10(0.7)$ \\
PT 5b & $83(6.2)$ & PT 22 & $9(0.7)$ \\
PT 4 & $76(5.7)$ & PT 23 & $8(0.6)$ \\
PT 6a & $49(3.7)$ & PT 19 & $7(0.5)$ \\
atypical & $33(2.5)$ & PT 51 & $6(0.4)$ \\
PT 6 & $20(1.5)$ & other & $57(4.3)$ \\
\hline
\end{tabular}

$n=$ number of $S$. Enteritidis cases with the phage type. $N=$ total number of $S$. Enteritidis cases that were phage typed during the 2 -year study period $=1,336$ highest IRs observed in the Central West and Central East regions. For PT $5 \mathrm{~b}$, the IR ranged from 0.19 to 0.58 units (mean $=0.31$, with the highest IRs observed in the Central West and North East regions. For PT 8, the IR ranged from 0.79 to 4.57 units (mean $=1.84$ ), with the highest IRs observed in the North West and Toronto regions. For PT 13, the IR ranged from 0.35 to 1.39 units (mean $=0.75)$, with the highest IRs observed in the Toronto and North West regions. For PT 13a, the IR ranged from 0 to 1.18 units (mean $=0.74$ ), with the highest IRs observed in the Toronto and Eastern regions.

\section{Monthly raw and smoothed incidence rates}

Time-series of raw and smoothed IRs of $S$. Enteritidis infections per 100,000 person-months for the six most frequent phage types in Ontario are illustrated in Fig. 4, and are described below. The monthly IR ranged from 0 to 0.14 units (mean $=0.04$ ) for PT 1,0 to 0.09 units $($ mean $=0.02)$ for PT 4,0 to 0.08 units (mean $=0.03)$ for PT 5b, 0.05 to 0.20 units (mean $=0.13$ ) for PT 8,0 to 0.12 units (mean $=0.06$ ) for PT 13 , and 0 to 0.16 units $($ mean $=0.07)$ for PT 13a.

Visually assessing the smoothed trend lines, a number of patterns were observed (Fig. 4). For PT 1, there were steep up slopes and gradual down slopes, with peaks occurring in January 2008, May 2009, and December 2009. For PT 4, there were three small peaks, which occurred in February 2008, November 2008, and March 2009. For PT 5b, there was one high peak in January 2009. For PT 8 , there were monthly variations with five peaks, which occurred in February 2008, November 2008, February 2009, May 2009, and October 2009. For PT 13, there were two peaks, which occurred in May 2008 and May 2009. For PT 13a, there was a high plateau between August 2008 and October 2008, a small plateau between August 2009 and October 2009, and a small peak in December 2009.

\section{Clinical symptoms}

Of the $1,336 S$. Enteritidis cases with known phage types, 1,123 cases $(84.1 \%)$ had clinical symptom information available. The most commonly reported symptoms were diarrhea (89-97\% of cases depending on the phage type), abdominal pain (49-64\%), fever (43-55\%), vomiting (23-33\%), and nausea (28-36 \%) (Table 2).

\section{Exposure settings}

Of the $1,336 \mathrm{~S}$. Enteritidis cases with known phage types, $372(27.8 \%)$ cases were missing exposure setting information, leaving 964 cases $(72.2 \%)$ available for exposure setting analysis (Table 3). International travel $(19.7 \%$ of 1,336 cases), private home $(7.0 \%)$, and food premise (6.4 \%) were the most commonly reported known 

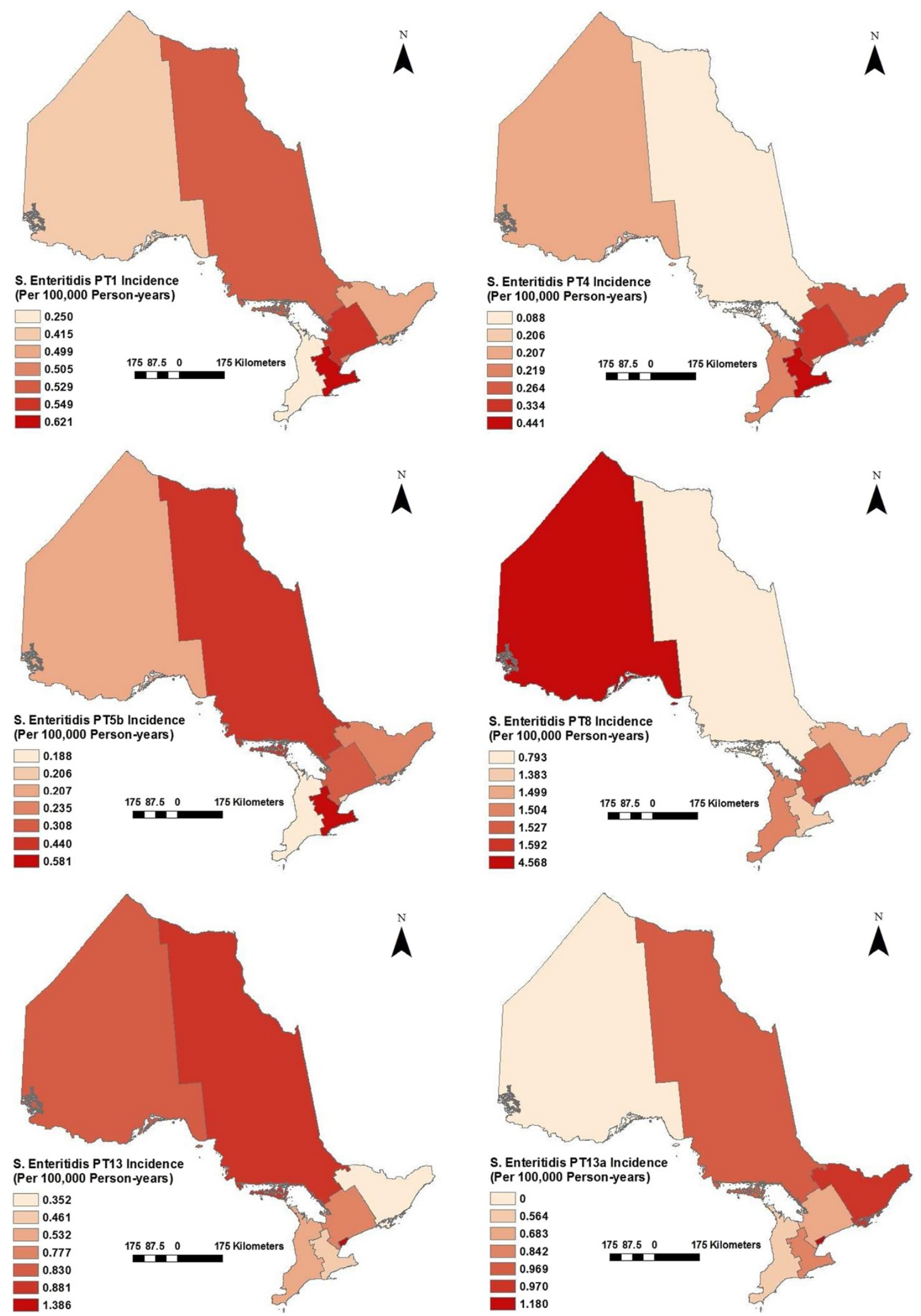

Fig. 3 Health region-level raw incidence rates of Salmonella Enteritidis cases with major phage types in Ontario, Canada, 2008-2009 


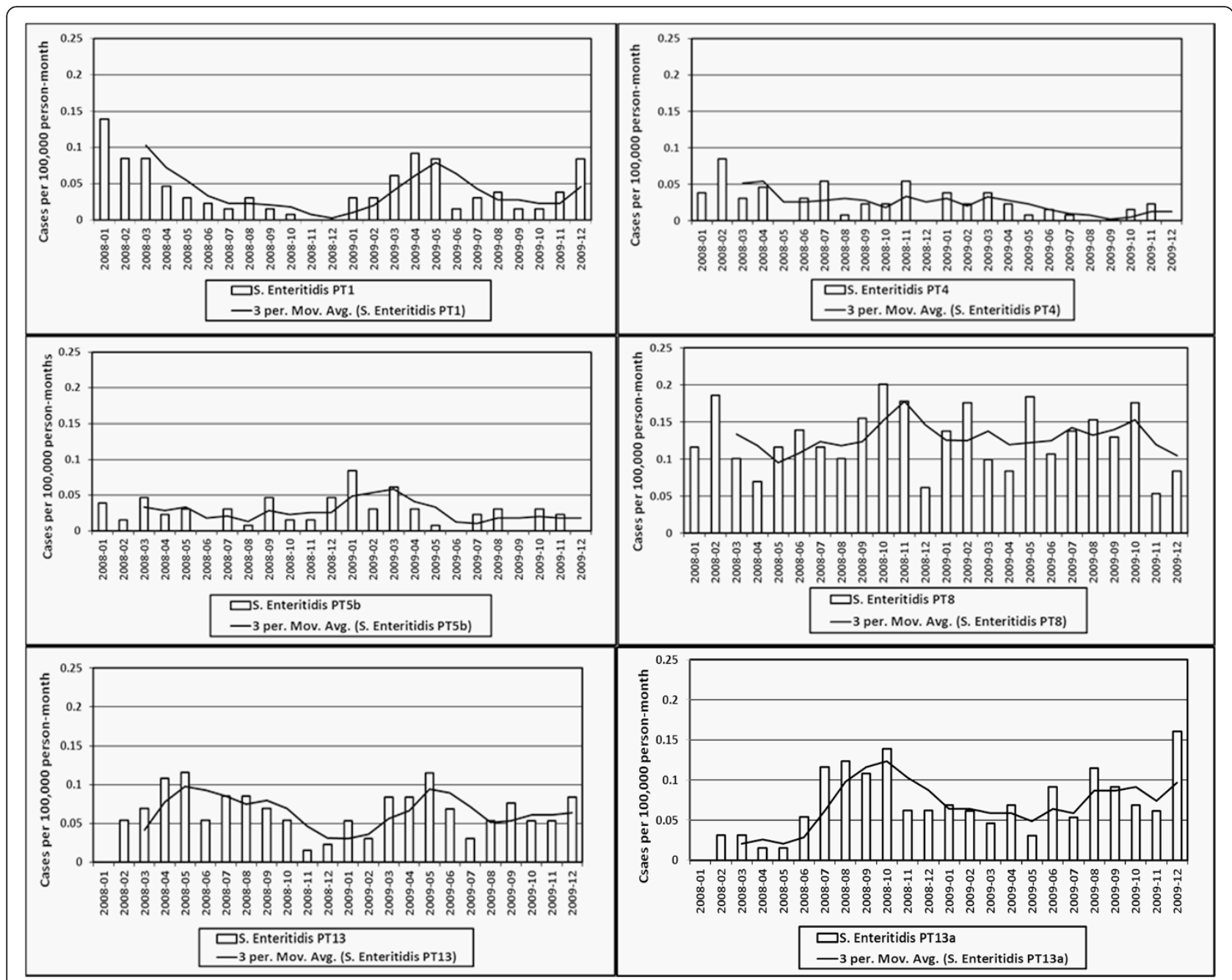

Fig. 4 Monthly raw and smoothed incidence rates of Salmonella Enteritidis cases with major phage types in Ontario. Smoothed IRs were based on a 3-month simple rolling average

Table 2 Clinical symptoms of Salmonella Enteritidis cases with major phage types in Ontario, Canada, 2008-2009 (N=1,123)

\begin{tabular}{|c|c|c|c|c|c|c|}
\hline \multirow[t]{3}{*}{ Phage Type } & \multirow{3}{*}{$\begin{array}{l}\text { Diarrhea } \\
\text { n (n/N \%) }\end{array}$} & \multicolumn{4}{|l|}{ Symptom } & \multirow{3}{*}{$\begin{array}{l}\text { Nausea } \\
\text { n (n/N \%) }\end{array}$} \\
\hline & & Bloody diarrhea & Abdominal pain & Fever & Vomiting & \\
\hline & & $\mathrm{n}(\mathrm{n} / \mathrm{N} \%)$ & $n(n / N \%)$ & n (n/N \%) & n (n/N \%) & \\
\hline PT $1(N=114)$ & $106(93)$ & $3 k$ & $67(59)$ & $49(43)$ & $34(30)$ & $36(32)$ \\
\hline PT $4(N=63)$ & $61(97)$ & $3(5)$ & $38(60)$ & $33(52)$ & $21(33)$ & $19(30)$ \\
\hline PT $5 b(N=75)$ & $70(93)$ & $5(7)$ & $37(49)$ & $36(48)$ & $17(23)$ & $27(36)$ \\
\hline PT $8(N=333)$ & $315(95)$ & $33(10)$ & $213(64)$ & $178(54)$ & $96(29)$ & $95(29)$ \\
\hline PT $13(N=169)$ & $150(89)$ & $18(11)$ & $95(56)$ & $86(51)$ & $53(31)$ & $33(20)$ \\
\hline PT 13a $(N=178)$ & $171(96)$ & $19(11)$ & $111(62)$ & $96(54)$ & $48(30)$ & $49(28)$ \\
\hline PT Others $(N=191)$ & $180(94)$ & $12(6)$ & $117(61)$ & $105(55)$ & $59(31)$ & $62(32)$ \\
\hline All PTs $(N=1,123)$ & $1,053(94)$ & $93(8)$ & $678(60)$ & $583(52)$ & $328(29)$ & $321(29)$ \\
\hline
\end{tabular}

Of the 1,336 S. Enteritidis cases with known phage types, 1,123 cases had clinical symptom information available. $n=$ number of $S$. Enteritidis cases that had the symptom. $N=$ number of $S$. Enteritidis cases with the phage type. Within a row, the percentages can add up to greater than $100 \%$ because a case could have more than one symptom 
Table 3 Exposure settings of Salmonella Enteritidis cases with major phage types in Ontario, Canada, 2008-2009

\begin{tabular}{|c|c|c|c|c|c|c|}
\hline \multirow[t]{3}{*}{ Phage type } & \multicolumn{4}{|c|}{ Exposure setting } & \multirow{3}{*}{$\begin{array}{l}\text { Unknown } \\
\text { n (n/M \%) }\end{array}$} & \multirow{3}{*}{$\begin{array}{l}\text { Missing } \\
(\mathrm{n} / \mathrm{N} \%)\end{array}$} \\
\hline & Private home & Food premise & International travel & Other setting & & \\
\hline & n (n/M \%) & n (n/M \%) & n (n/M \%) & n (n/M \%) & & \\
\hline PT $1(N=132)(M=93)$ & $3(3)$ & $4(4)$ & $60(65)$ & 0 & $26(28)$ & $39(30)$ \\
\hline PT $4(N=76)(M=48)$ & 0 & $3(6)$ & $33(69)$ & $1(2)$ & $11(23)$ & $28(37)$ \\
\hline PT $5 b(N=83)(M=58)$ & 0 & $1(2)$ & $44(76)$ & 0 & $13(22)$ & $25(30)$ \\
\hline PT $8(N=398)(M=285)$ & $38(13)$ & $33(12)$ & $29(10)$ & $4(1)$ & $181(64)$ & $113(28)$ \\
\hline PT $13(N=198)(M=158)$ & $21(13)$ & $24(15)$ & $10(6)$ & $3(2)$ & $100(64)$ & $40(20)$ \\
\hline PT 13a $(N=218)(M=157)$ & $22(14)$ & $14(9)$ & $14(9)$ & $4(2)$ & $103(66)$ & $61(28)$ \\
\hline PT Others $(N=231)(M=165)$ & $9(6)$ & $7(4)$ & $73(44)$ & $4(2)$ & $72(44)$ & $66(29)$ \\
\hline Total $(N=1336)(M=964)$ & $93(10)$ & $86(9)$ & $263(27)$ & $16(2)$ & $506(52)$ & $372(28)$ \\
\hline
\end{tabular}

Of the 1,336 S. Enteritidis cases with known phage types, 964 cases had exposure setting information available. $n=$ number of $S$. Enteritidis cases with the exposure setting. $M=$ number of $S$. Enteritidis cases that had exposure setting information available. $N=$ number of $S$. Enteritidis cases with the phage type. Exposure settings in the surveillance database were categorized as: international travel (i.e., travelled outside of Canada), private home, food premise (e.g., restaurant, grocery store, bakery, deli, caterer, mobile food premise), other (e.g., institution, hospital, farm, petting zoo, child care centre), or unknown (if the only exposure reported was "unknown")

exposure settings. Unknown exposure setting was reported for $37.9 \%$ of cases.

Of the six most frequent $S$. Enteritidis phage types $(n=1,105), 306(27.7 \%)$ cases were missing exposure setting information, leaving 799 (72.3 \%) cases available for exposure setting analysis. Unknown exposure setting was reported for 434 (39.3\%) of cases. Known exposure setting information was reported for 365 (33.03 \%) of cases: 67 PT 1 cases (50.8\% of all PT 1 cases), 37 PT 4 cases (48.7\% of all PT 4 cases),

Table 4 Clusters of Salmonella Enteritidis cases with the six most frequent phage types in Ontario, Canada, 2008-2009

\begin{tabular}{|c|c|c|c|c|c|c|c|c|c|}
\hline Phage type $(\mathrm{N})$ & $\begin{array}{l}\text { Annual cases per } \\
100,000\end{array}$ & $\begin{array}{l}\text { Cluster } \\
\text { type }\end{array}$ & Region & $\begin{array}{l}\text { Time frame (Year/ } \\
\text { Month) }\end{array}$ & Observed & Expected & O/E & $R R$ & $\begin{array}{l}P- \\
\text { value }\end{array}$ \\
\hline \multirow[t]{3}{*}{ PT $1(N=132)$} & \multirow[t]{3}{*}{0.05} & \multirow[t]{2}{*}{$\begin{array}{l}\text { Space- } \\
\text { time }\end{array}$} & $\begin{array}{l}\text { North West, North East, Eastern, } \\
\text { Central East }\end{array}$ & $2008 / 1$ to $2008 / 2$ & 20 & 5.17 & 3.87 & 4.38 & $\leq 0.001$ \\
\hline & & & Central West & $2008 / 1$ to $2008 / 5$ & 16 & 5.24 & 3.05 & 3.33 & 0.046 \\
\hline & & Temporal & All & $2008 / 1$ to $2008 / 3$ & 40 & 16.36 & 2.38 & 3.07 & 0.001 \\
\hline \multirow[t]{2}{*}{ PT $4(N=76)$} & \multirow[t]{2}{*}{0.03} & $\begin{array}{l}\text { Space- } \\
\text { time }\end{array}$ & Eastern, Central East & $2008 / 2$ to $2008 / 4$ & 15 & 3.89 & 3.85 & 4.55 & 0.010 \\
\hline & & Temporal & All & $2008 / 1$ to $2008 / 11$ & 51 & 34.67 & 1.47 & 2.43 & 0.009 \\
\hline \multirow[t]{3}{*}{ PT $5 b(N=83)$} & \multirow[t]{3}{*}{0.03} & Spatial & Central West & NA & 29 & 15.93 & 1.82 & 2.26 & 0.003 \\
\hline & & $\begin{array}{l}\text { Space- } \\
\text { time }\end{array}$ & Central West & $2008 / 9$ to $2009 / 4$ & 17 & 5.27 & 3.22 & 3.80 & 0.016 \\
\hline & & Temporal & All & $2008 / 12$ to $2009 / 3$ & 29 & 13.77 & 2.11 & 2.70 & 0.002 \\
\hline \multirow[t]{3}{*}{ PT $8(N=397)$} & \multirow[t]{3}{*}{0.20} & Spatial & North West & NA & 22 & 7.37 & 2.98 & 3.10 & $\leq 0.001$ \\
\hline & & \multirow{2}{*}{$\begin{array}{l}\text { Space- } \\
\text { time }\end{array}$} & North West & $2009 / 2$ to $2009 / 5$ & 15 & 1.20 & 12.46 & 12.91 & $\leq 0.00$ \\
\hline & & & South West & $2008 / 9$ to $2008 / 12$ & 21 & 8.11 & 2.59 & 2.68 & 0.046 \\
\hline \multirow[t]{3}{*}{ PT $13(N=198)$} & \multirow[t]{3}{*}{0.08} & Spatial & Toronto & NA & 74 & 40.71 & 1.82 & 2.31 & $\leq 0.001$ \\
\hline & & $\begin{array}{l}\text { Space- } \\
\text { time }\end{array}$ & Toronto & $2008 / 4$ to $2008 / 10$ & 40 & 11.87 & 3.37 & 3.97 & $\leq 0.00$ \\
\hline & & Temporal & All & $2008 / 4$ to $2008 / 5$ & 29 & 16.46 & 1.76 & 1.89 & 0.051 \\
\hline \multirow[t]{3}{*}{ PT 13a $(N=218)$} & \multirow[t]{3}{*}{0.08} & Spatial & Toronto & NA & 63 & 44.70 & 1.41 & 1.58 & 0.018 \\
\hline & & $\begin{array}{l}\text { Space- } \\
\text { time }\end{array}$ & Toronto & $\begin{array}{l}2009 / 10 \text { to } 2009 / \\
12\end{array}$ & 18 & 5.64 & 3.19 & 3.39 & 0.018 \\
\hline & & Temporal & All & $2008 / 7$ to $2008 / 10$ & 63 & 36.51 & 1.73 & 2.02 & 0.001 \\
\hline
\end{tabular}

$N=$ number of $S$. Enteritidis cases with the phage type. Results based on discrete Poisson models using the SaTScan ${ }^{\mathrm{TM}}$ software. Study period: January 1,2008 to December 31, 2009. Time aggregation units: month. Time aggregation length: 1 month. Circular scanning window size: up to $50 \%$ of the population at risk and/ or $50 \%$ of time the study period. Confounders controlled for: age $(0-9,10-24,25-34,35-49, \geq 50$ years) and sex. Criteria for reporting secondary clusters: no geographical overlap. Type of clusters investigated: high rate only. NA $=$ not applicable. $\mathrm{O} / \mathrm{E}=$ observed divided by expected. $\mathrm{RR}=$ relative risk. Significance level: $p \leq 0.05$ 
45 PT $5 \mathrm{~b}$ cases (54.2\% of all PT 5b cases), 104 PT 8 cases (26.2\% of all PT 8 cases), 58 PT 13 cases (29.3\% of all PT 13 cases), and 54 PT 13a cases (24.8\% of all PT 13a cases).

Of the cases that had exposure setting information available, international travel and unknown exposure settings were the most frequently reported settings for PT $5 \mathrm{~b}$ cases (76 and $22 \%$ of PT $5 \mathrm{~b}$ cases, respectively), PT 4 cases (69 and $23 \%$ of PT 4 cases, respectively), and PT 1 cases (65 and $28 \%$ of PT 1 cases, respectively). Unknown, private home, food premise, and international travel were the most frequently reported exposure settings for PT 8 cases $(64,13,12$, and $10 \%$ of PT 8 cases, respectively), PT 13 cases $(64,13,15$, and $6 \%$ of PT 13 cases, respectively), and PT 13 a cases $(66,14,9$, and $9 \%$ of PT 13a cases, respectively) (Table 3 ).

\section{Scan statistics}

\section{Purely spatial clusters of S. Enteritidis cases}

Four significant high rate spatial clusters were detected (Table 4 and Fig. 5). A cluster of 29 PT 5b cases was identified in the Central West region $(\mathrm{RR}=2.26, p=0.003)$. A

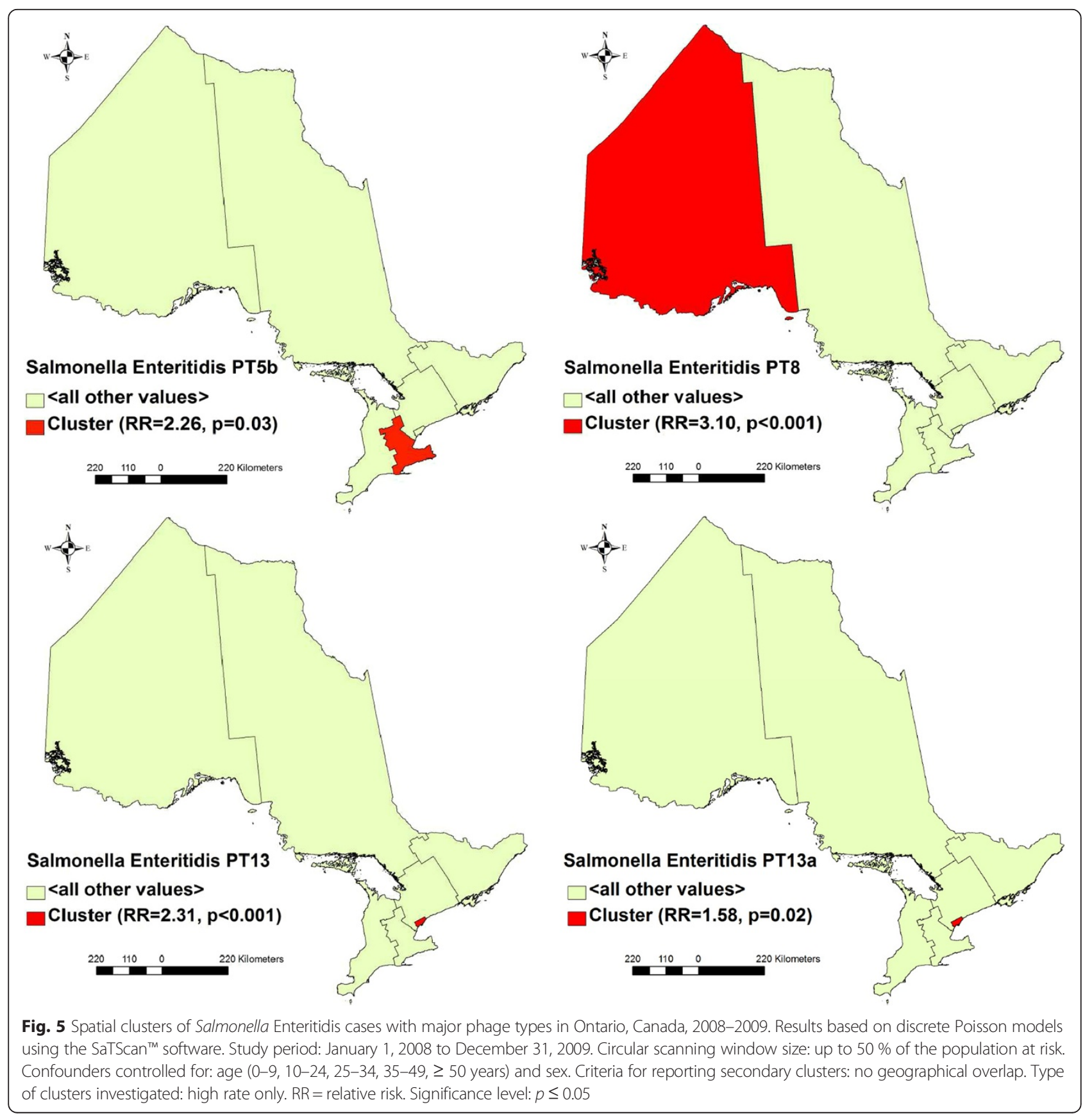




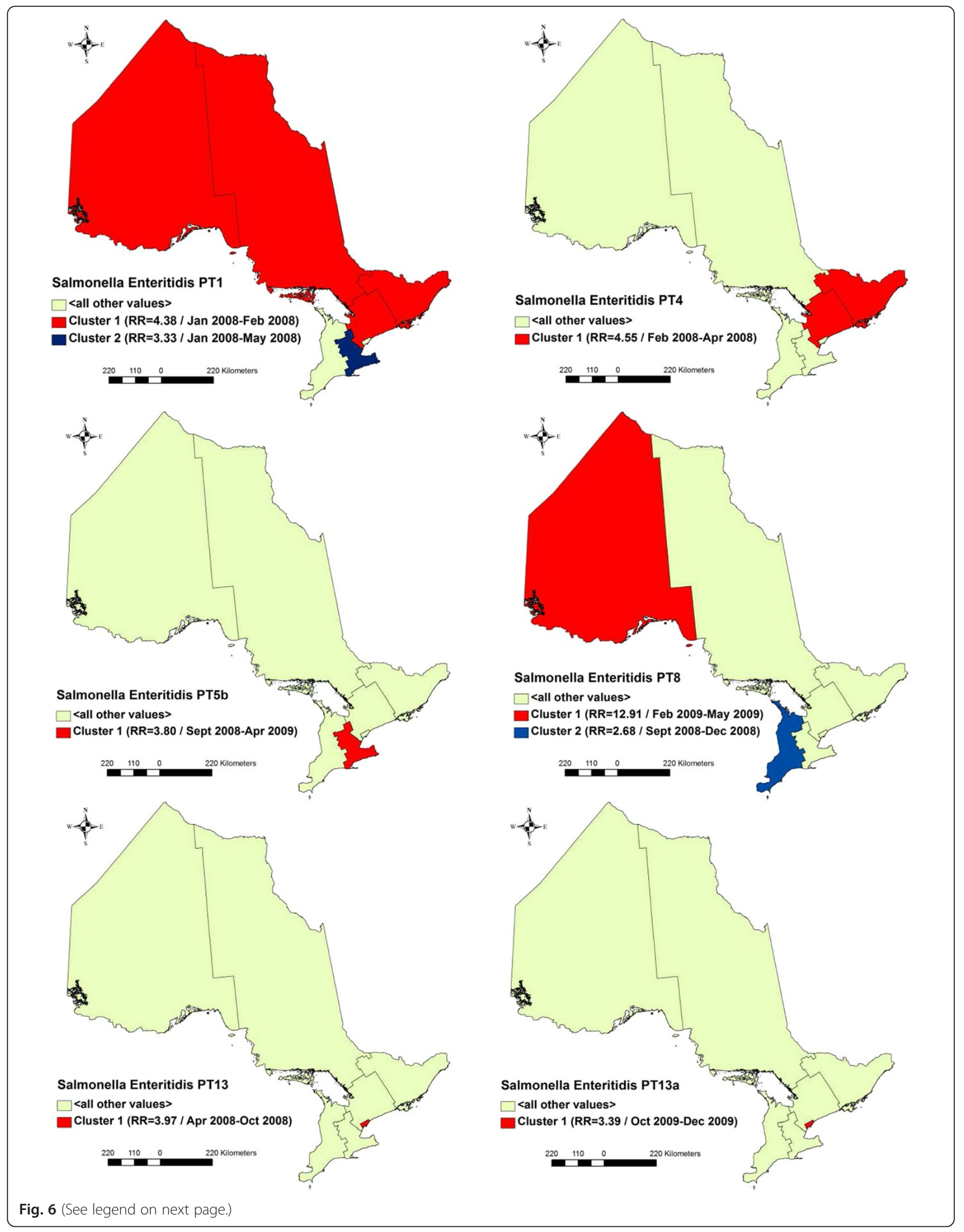


(See figure on previous page.)

Fig. 6 Space-time clusters of Salmonella Enteritidis cases with major phage types in Ontario, Canada, 2008-2009. Results based on discrete Poisson models using the SaTScan ${ }^{\mathrm{TM}}$ software. Study period: January 1, 2008 to December 31, 2009. Circular scanning window size: up to $50 \%$ of the population at risk and $50 \%$ of the study period. Confounders controlled for: age $(0-9,10-24,25-34,35-49$, $\geq 50$ years) and sex. Time aggregation units: month. Time aggregation length: 1 month. Criteria for reporting secondary clusters: no geographical overlap. Type of clusters investigated: high rate only. RR = relative risk. Significance level: $p \leq 0.05$

cluster of 22 PT 8 cases was identified in the North West region ( $R R=3.10, p \leq 0.001)$. A cluster of 74 PT 13 cases was identified in the Toronto region $(\mathrm{RR}=2.31, p \leq 0.001)$. A cluster of 63 PT 13a cases was identified in the Toronto region $(\mathrm{RR}=1.58, p=0.018)$.

\section{Purely temporal clusters of S. Enteritidis cases}

Five significant high rate temporal clusters were detected (Table 4). A cluster of 40 PT 1 cases occurred from January to March $2008(\mathrm{RR}=3.07, p=0.001)$. A cluster of $51 \mathrm{PT} 4$ cases occurred from January to November 2008 ( $R R=2.43$, $p=0.009)$. A cluster of $29 \mathrm{PT} 5 \mathrm{~b}$ cases occurred from December 2008 to March $2009(\mathrm{RR}=2.70, p=0.002)$. A cluster slightly above the rejection threshold of $29 \mathrm{PT}$ 13 cases occurred from April to May 2008 ( $R R=1.89$, $p=0.051)$. A cluster of 63 PT 13 a cases occurred from July to October $2008(\mathrm{RR}=2.02, p=0.001)$.

\section{Space-time clusters of $S$. Enteritidis cases}

Eight significant high rate space-time clusters were detected, including two secondary clusters (Table 4 and Fig. 6). Two clusters of PT1 cases were identified: a primary cluster of 20 cases occurred from January to February 2008 in the North West, North East, Eastern, and Central East regions $(\mathrm{RR}=4.38, p \leq 0.001)$; and a secondary cluster of 16 cases occurred from January to May 2008 in the Central West region $(\mathrm{RR}=3.33, p=0.046)$. A cluster of $15 \mathrm{PT} 4$ cases occurred from February to April 2008 in the Eastern and Central East regions $(\mathrm{RR}=4.55, p=0.010)$. A cluster of 17 PT 5b cases occurred from September 2008 to April 2009 in the Central West region ( $\mathrm{RR}=3.80, p=0.016)$. Two clusters of PT 8 cases were identified: a primary cluster of 15 cases occurred from February to May 2009 in the North West region $(\mathrm{RR}=12.91, p \leq 0.001)$; and a secondary cluster of 21 cases occurred from September to December 2008 in the South West region ( $R R=2.68, p=0.046)$. A cluster of 40 PT 13 cases occurred from April to October 2008 in the Toronto region $(\mathrm{RR}=3.97, p \leq 0.001)$. A cluster of $18 \mathrm{PT}$ 13a cases occurred from October to December 2009 in the Toronto region $(\mathrm{RR}=3.39, p=0.018)$.

\section{Space-time cluster cases' exposure settings}

Exposure setting information was unknown or missing for many of the cases that were part of the space-time clusters (Table 5). For the primary PT 1 cluster, exposure setting information was known for 9 of the 20 cases; seven cases reported international travel and two cases reported food premises as their exposure setting. For the secondary PT 1 cluster, exposure setting information was known for 4 of the 16 cases; all four cases reported international travel as their exposure setting. For the PT 4 cluster, exposure setting information was known for 9 of the 15 cases; all nine cases reported international travel as their exposure setting. For the PT $5 \mathrm{~b}$ cluster, exposure setting information was known for 9 of the 17 cases; all nine cases reported international travel as their exposure setting. For the primary PT 8 cluster, no exposure setting information was known for the 15 cases. For the secondary PT 8 cluster, exposure setting information was known for 7 of the 21 cases; three cases reported food premises, two cases reported private homes, one case reported other setting, and one case reported international travel as their exposure setting. For the PT 13 cluster, exposure setting information was known for 14 of the 40 cases; 10 cases reported food premises, two cases reported private homes, one case reported other setting, and one case reported international travel as their exposure setting. For the PT 13a cluster, exposure setting information was known for 11 of the 18 cases; seven cases reported food premises, three cases reported international travel, and one case reported private home as their exposure setting.

\section{Discussion}

Our study enhanced the current knowledge on the spatial and temporal epidemiology of the phage types of $S$. Enteritidis that predominate in Ontario health regions. We used a step-wise approach, starting with a general exploratory analysis followed by a more specific statistical analysis. A number of phage type-specific high rate areas and time periods were identified during the exploratory analysis that were confirmed by the statistical analysis as significant spatial, temporal, or space-time clusters of cases.

Foodborne disease clusters are generally defined as the occurrence of a higher than expected number of cases for a given location and/or time period. These clusters may or may not meet the definition of an outbreak [32, 33]. Subtype-based surveillance systems frequently use the term "cluster" to describe a group of cases infected with identical microbial strains [32]. Subtyping is useful for differentiating between endemic and outbreak cases, especially for common Salmonella serotypes, such as Enteritidis, that occur sporadically throughout the year 
Table 5 Exposure settings of the Salmonella Enteritidis cases included in the space-time clusters for the six most frequent phage types in Ontario, Canada, 2008-2009

\begin{tabular}{|c|c|c|c|c|c|c|c|c|}
\hline \multicolumn{3}{|c|}{ Phage type Cluster } & \multicolumn{5}{|c|}{ Exposure setting } & \multirow[b]{2}{*}{$\begin{array}{l}\text { Missing } \\
(\mathrm{n})\end{array}$} \\
\hline Phage type (N) & Region & $\begin{array}{l}\text { Cases } \\
\text { (n) }\end{array}$ & $\begin{array}{l}\text { Private home } \\
\text { (n) }\end{array}$ & $\begin{array}{l}\text { Food } \\
\text { premise (n) }\end{array}$ & $\begin{array}{l}\text { International } \\
\text { travel }(\mathrm{n})\end{array}$ & $\begin{array}{l}\text { Other setting } \\
\text { (n) }\end{array}$ & $\begin{array}{l}\text { Unknown } \\
\text { (n) }\end{array}$ & \\
\hline \multirow[t]{2}{*}{ PT $1(N=132)$} & $\begin{array}{l}\text { North West, North East, Eastern, } \\
\text { Central East }\end{array}$ & 20 & 0 & 2 & 7 & 0 & 4 & 7 \\
\hline & Central West & 16 & 0 & 0 & 4 & 0 & 3 & 9 \\
\hline PT $4(N=76)$ & Eastern, Central East & 15 & 0 & 0 & 9 & 0 & 1 & 5 \\
\hline PT $5 b(N=83)$ & Central West & 17 & 0 & 0 & 9 & 0 & 4 & 4 \\
\hline \multirow[t]{2}{*}{ PT $8(N=397)$} & North West & 15 & 0 & 0 & 0 & 0 & 13 & 2 \\
\hline & South West & 21 & 2 & 3 & 1 & 1 & 5 & 9 \\
\hline PT $13(N=198)$ & Toronto & 40 & 2 & 10 & 1 & 1 & 26 & 0 \\
\hline PT 13a $(N=218)$ & Toronto & 18 & 1 & 7 & 3 & 0 & 7 & 0 \\
\hline
\end{tabular}

$N=$ number of $S$. Enteritidis cases with the phage type. $n=$ number of $S$. Enteritidis cases with the exposure setting. Results based on discrete Poisson models using the SatTScan ${ }^{T M}$ software. Study period: January 1, 2008 to December 31, 2009. Time aggregation units: month. Time aggregation length: 1 month. Circular scanning window size: up to $50 \%$ of the population at risk and $50 \%$ of the study period. Confounders controlled for age (0-9, 10-24, 25-34, 35-49, $\geq 50$ years) and sex. Criteria for reporting secondary clusters: no geographical overlap. Type of clusters investigated: high rate only. Exposure settings in the surveillance database were categorized as: international travel (i.e., travelled outside of Canada), private home, food premise (e.g., restaurant, grocery store, bakery, deli, caterer, mobile food premise), or other (e.g., institution, hospital, farm, petting zoo, child care centre)

[34]. Differences in reservoirs and exposure settings might exist for different $S$. Enteritidis phage types, and molecular differentiation can help to understand potential sources of the different phage types $[8,34]$. We defined a cluster as a health region, time period, or a health region during a particular time period with a statistically significant higher than expected phage type-specific $S$. Enteritidis infection rate. Thus, we demonstrated the effectiveness of using cluster detection tests, in conjunction with subtyping methods to understand the epidemiology of a foodborne pathogen.

A number of patterns were observed when assessing the geographical heterogeneity of health region-level IRs of $S$. Enteritidis infections for the most frequent phage types. The Central West region had the highest IRs for PTs 1,4 , and $5 \mathrm{~b}$, whereas the Toronto region had the highest IRs for PTs 13 and 13a. Several of these regions were later confirmed by the spatial scan statistic as regions with significant high rate clusters (e.g., cases of PT $5 \mathrm{~b}$ significantly clustered in the Central West region and cases of PTs 13 and 13a significantly clustered in the Toronto region).

We used a smoothing method for our time-series graph to reduce the month-to-month random variation of infection rates and make the overall trends clearer. The observed trends were relatively consistent with the results of the purely temporal scan statistic, albeit not as definitive. With the exception of PT 5b, all temporal clusters occurred during 2008. Further, most clusters occurred during a distinct season. Cases of PTs 1 and 5b clustered during the winter months, cases of PT 13 clustered during the spring months, and cases of PT 13a clustered during the summer and fall months.
Differences in the duration of the temporal clusters were also observed. The majority of clusters (PTs 1, 5b, 13, and $13 \mathrm{a})$ were of relatively short duration (2-4 months), whereas the PT 4 cluster was of long duration (11 months). Of note, the most commonly reported phage type (PT 8) did not cluster temporally, suggesting a fairly even distribution of PT 8 cases over time throughout Ontario. A study conducted in Alberta, Canada, examining Salmonella serotypes rather than phage types, detected several serotype-specific temporal clusters during the 11-year study period (January 1990 to January 2002) [35]; for $S$. Enteritidis, the clusters were of short duration and occurred during the winter and spring months.

The exposure setting information is rarely confirmed by data obtained through environmental health investigations or statistical associations obtained through casecontrol or cohort studies [36]; however, it is considered to be useful epidemiological data for foodborne illness source attribution [37]. Knowing when, where, and why clusters occurred can aid in the development of effective outbreak detection, prevention, and control programs. Our study identified differences between phage types with respect to the time and duration of the space-time clusters, even for clusters occurring in the same region. For example, the PT 13 and 13a clusters both occurred in the Toronto region, but during different time periods (the cluster of cases with PT 13 occurred in 2008, whereas the cluster of cases with PT 13a occurred in 2009). Moreover, the cluster of cases with PT 13 was of long duration (7 months), whereas the cluster of cases with PT 13a was of short duration (3 months). Short duration clusters might signify that cases were exposed 
to a single infection source (e.g., point source outbreak). Long duration clusters might signify that cases were exposed to a single source (e.g., contaminated food) over a longer time period (e.g., continuous common source outbreak) [32, 33, 35], to multiple sources (e.g., continuous multiple source outbreak) [32,33], to the occurrence of secondary infections [35], to poor food preparation practices over a prolonged period, or that the typing method used was not of high enough resolution to differentiate between different strains.

Many of the cases with PT 13 or 13a that were part of a space-time cluster reported food premises (e.g., restaurant, grocery store, bakery, deli, caterer, mobile food premise) as their main exposure setting. In North America, restaurants have been shown to be an important exposure setting for $S$. Enteritidis infections [38-41]. A number of predisposing factors for food contamination with $S$. Enteritidis in restaurants were identified, including cross contamination from raw chicken meat to food server's hands or cutting boards due to high food volumes and food handler's improper food safety practices during food preparation [36, 38], inadequate heat treatment of foods [38], inappropriate food storage [38], and direct contamination of food served by infected food handlers $[10,39-41]$. In Ontario, $S$. Enteritidis accounted for only $10.1 \%$ of the Salmonella isolates collected at pre-harvest from conventionally-raised broiler chicken flocks between July 2010 and April 2012; 65 \% of the isolates were PT 13a (Tara Roberts, 2014, personal communication).

A few of the cases that were part of a PT 8, 13, or 13a space-time cluster reported private homes as their exposure setting. Previous studies identified private homes as an important exposure setting for sporadic, homebased foodborne infections [42-44]. Several predisposing factors of home-based infections have been identified, including inappropriate food handling, storage, and food preparation [42, 43]; consumption of contaminated raw and undercooked foods [42]; and person-to-person [44] and animal-to-person $[45,46]$ transmission.

Space-time clusters of cases with PT 1 or 4 included several overlapping health regions, occurred during nearly identical winter and spring months, and were of short duration (2-3 months). The majority of these cases reported international travel as their exposure setting. International travel was demonstrated by a number of studies as an important risk factor for $S$. Enteritidis infections in North America [15, 47, 48]. In the US, among all salmonellosis cases between 2004 and 2008, $11 \%$ reported international travel as their exposure setting, and among those, the most commonly reported serotype was Enteritidis (22\% of travel cases) [47]. In the region of Waterloo, Ontario, Canada, between June 2005 and May 2009, $48.7 \%$ of $S$. Enteritidis cases were international travel-related [48]. In Ontario, Canada, between July 2010 and June 2011, $51.9 \%$ of S. Enteritidis infections were international travel-related, and certain phage types (e.g., 1, 4, and 5b) were isolated from cases who visited all-inclusive resorts in the Caribbean or Mexico during the winter and spring months [15]. The seasonal spike of PT 1 and 4 cases in late winter and early spring, when people often travel to warmer destinations, warrants creating advisories to inform travelers about the risks of eating abroad and how they can protect themselves against $S$. Enteritidis infections.

A number of limitations should be recognized before interpreting our study results. Surveillance programs underestimate the true burden of infections due to underdiagnosis and under-reporting of cases [3]. In Canada, it was estimated that for every reported salmonellosis case there were 26.1 unreported cases in the general population [3]. Under-reporting and under-diagnosis can be influenced by differences in populations' medical care seeking behaviour and access to medical care [49], physicians' specimen request and diagnosis practices [50], and laboratories testing protocols and reporting standards [50]. Regional differences in successful case follow-up should also be considered. Loss to follow-up of cases might be greater in low population density regions of the province due to difficulties encountered by public health staff in contacting cases. A large number of cases had missing or unknown exposure setting information, which might have biased our study results. The proportion and accuracy of known exposure setting information reported by investigators can depend on several factors [36], including time passed from exposure to case interview and the related recall bias, difficulty and the effort made by the investigator to contact a case, follow-up protocol and questionnaire used by the investigator (e.g., face to face interview vs. phone interview vs. questionnaire sent through the mail), a case's willingness to be interviewed, and possible survival bias. In our study, differences in unknown exposure setting among phage types were noted. The proportion of unknown exposure setting information was higher for cases with PT 8, 13, or 13a (64-66 \%) compared to cases with PT 1,4 , or $5 \mathrm{~b}(22-28 \%)$, suggesting that international travel cases had more readily available exposure history; therefore in our study, the overall proportion of cases who reported international travel as their major exposure setting was likely slightly over-estimated. Lastly, misclassification of international travel-related cases might have also occurred, especially for cases for which the incubation period was short, and for cases with a longer disease incubation period who became infected before departure [48].

Obtaining exposure setting information is a first step toward developing effective prevention and control programs; however, the location and the primary source of 
contamination of food products that lead to infections are not always identical [51-52]. Therefore, future research studies are needed to identify the primary source of contamination, and the type of food products that cause infections.

This study demonstrated the utility of retrospective spatial and temporal analysis of subtype-based surveillance data using exploratory and statistical methods to detect clusters of cases. Phage type-specific spatial and spatial-temporal clusters should be followed up by public health authorities to identify novel local individuallevel risk factors. Increased enforcement (e.g., restaurant inspections) and education (e.g., food safety training for restaurant employees and the general public) in health regions with identified spatial or spatial-temporal clusters have the potential to decrease the incidence of PTs 8, 13, and 13a. Further, prevention programs (e.g., travel advisories) that are targeted during the winter and spring months have the potential to decrease the incidence of PTs 1,4 , and $5 \mathrm{~b}$. During the study period no outbreaks were reported in Ontario; thus, the evaluation of current outbreak detection methods used by public health staff at various PHUs is warranted. Future studies are needed to evaluate the frequency of false positive clusters, to assess the effectiveness of cluster detection using statistical methods, to compare the more traditional outbreak investigation procedures to scan statistic cluster detection techniques, and to measure the feasibility of statistical methods for identifying infection clusters. Purely spatial or purely temporal clusters might be the result of a space-time cluster, which should be considered when evaluating our study results. There is a need also for prospective research studies to identify clusters of $S$. Enteritidis infections in real-time (e.g., weeks, months), and to assess and evaluate individual-level risk factors for infections included in these clusters. Moreover, there is a need for high resolution molecular subtyping methods (e.g., multiple locus variable-number tandem repeat analysis or whole genome sequencing) to better understand relationships between cases in a cluster.

\section{Conclusions}

This is the first study that has evaluated the spatial and temporal epidemiology of the phage types of $S$. Enteritidis that predominate in Ontario health regions. This study demonstrated the value of using a number of spatial-temporal and subtyping methods to better understand the epidemiology of a foodborne illness, such as salmonellosis. Our study highlighted phage type-specific differences in spatial distributions, temporal trends, clinical symptoms, exposure settings, and space-time clusters of $S$. Enteritidis infections. Several health regions were identified with increased phage type-specific $S$. Enteritidis infection rates where future studies should be conducted to identify novel individual-level risk factors, and where future prevention and control programs should be targeted to reduce the incidence of $S$. Enteritidis infections. Our study methodology may be applicable to other foodborne disease surveillance data.

\section{Additional file}

Additional file 1: Legend 1 for Fig. 1. Ontario Public Health Unit labels, names, and population estimates. (DOCX $16 \mathrm{~kb}$ )

\section{Abbreviations}

GIS: Geographic information system; iPHIS: Integrated Public Health Information System; IR: Incidence rate; MOHLTC: Ontario Ministry of Health and Long-Term Care; PHU: Public health unit; PT: Phage type; $S$.

Enteritidis: Salmonella enterica serovar Enteritidis; US: United States of America.

\section{Competing interests}

The authors declare that they have no competing interests.

\section{Authors' contributions}

CV developed the study design, analysed the data, interpreted results, wrote the first draft of the manuscript, responded to editorial comments, and prepared the final manuscript for submission. MTG was consulted on data analysis, study design, interpretation of results, and reviewed and commented on manuscript drafts. DLP, SAM, FP, and JMS provided advice on the data analysis, interpretation of results, and reviewed and commented on manuscript drafts. All authors read and approved the final manuscript.

\section{Acknowledgements}

The authors acknowledge the MOHLTC for providing the data. We thank the staff of all PHUs and public health laboratories that tested samples, followed up with cases, and entered information into the public health surveillance database. We also acknowledge the Data Resource Centre at the University of Guelph library for their GIS and census support. The views expressed in this study are the views of the authors and do not necessarily reflect those of the MOHLTC.

\section{Author details}

${ }^{1}$ Department of Population Medicine, Ontario Veterinary College, University of Guelph, Guelph, ON N1G 2W1, Canada. ${ }^{2}$ Ontario Ministry of Agriculture, Food and Rural Affairs, Guelph, ON N1G 4Y2, Canada. ${ }^{3}$ Centre for Public Health and Zoonoses, Ontario Veterinary College, University of Guelph, Guelph, ON N1G 2W1, Canada. ${ }^{4}$ Centre for Foodborne, Environmental and Zoonotic Infectious Diseases, Public Health Agency of Canada, Guelph, ON $\mathrm{N} 1 \mathrm{H}$ 8J1, Canada.

Received: 17 June 2015 Accepted: 9 December 2015

Published online: 17 December 2015

References

1. Majowicz SE, Musto J, Scallan E, Angulo FJ, Kirk M, O'Brien SJ, et al. The global burden of nontyphoidal Salmonella gastroenteritis. Clin Infect Dis. 2010;50(6):882-9.

2. Ruzante JM, Majowicz SE, Fazil A, Davidson VJ. Hospitalization and deaths for select enteric illnesses and associated sequelae in Canada, 2001-2004. Epidemiol Infect. 2011;139(6):937-45.

3. Thomas MK, Murray R, Flockhart L, Pintar K, Pollari F, Fazil A, et al. Estimates of the burden of foodborne illness in Canada for 30 specified pathogens and unspecified agents, circa 2006. Foodborne Pathog Dis. 2013;10(7):639-48.

4. Public Health Agency of Canada (PHAC). National Enteric Surveillance Program (NESP) Annual Summary 2010.Available at: http://publications.gc. ca/collections/collection_2012/aspc-phac/HP37-15-2010-eng.pdf

5. Centers for Disease Control and Prevention (CDC). Foodborne Diseases Active Surveillance Network (FoodNet). FoodNet Surveillance Report for 2011 (Final Report). Atlanta, Georgia: U.S. Department of Health and Human 
Services, CDC; 2012. Available at: http://www.cdc.gov/foodnet/PDFs/2011_ annual_report_508c.pdf.

6. Centers for Disease Control and Prevention (CDC). National Salmonella Surveillance Data: Salmonella Annual Summary Tables 2009. Atlanta, GA U.S. Department of Health and Human Services, CDC; 2008. Available at: http://www.cdc.gov/ncezid/dfwed/PDFs/

SalmonellaAnnualSummaryTables2009.pdf

7. European Food Safety Authority (EFSA). The Community Summary Report on trends and sources of zoonoses, zoonotic agents and food-borne outbreaks in the European Union in 2008. EFSA J. 2010;8(1):1496.

8. Varga C, Middleton D, Walton R, Savage R, Tighe MK, Allen V, et al. Evaluating risk factors for endemic human Salmonella Enteritidis infections with different phage types in Ontario. Canada using multinomial logistic regression and a case-case study approach. BMC Public Health. 2012;12:866.

9. Taylor M, Leslie M, Ritson M, Stone J, Cox W, Hoang L, et al. Investigation of the concurrent emergence of Salmonella enteritidis in humans and poultry in British Columbia, Canada, 2008-2010. Zoonoses Public Health. 2012;59(8): 584-92.

10. Centers for Disease Control and Prevention (CDC). Salmonella enteritidis infections associated with foods purchased from mobile lunch trucks Alberta, Canada, October 2010-February. MMWR Morb Mortal Wkly Rep 2013. 2011;62(28):567-9.

11. Marcus R, Varma JK, Medus C, Boothe EJ, Anderson BJ, Crume T, et al. Re-assessment of risk factors for sporadic Salmonella serotype Enteritidis infections: a case-control study in five FoodNet Sites, 2002-2003. Epidemiol Infect. 2007;135(1):84-92.

12. Lee SS, Wong NS. The clustering and transmission dynamics of pandemic influenza A (H1N1) 2009 cases in Hong Kong. J Infect. 2011; 63(4):274-80.

13. So HC, Pearl DL, von Königslöw T, Louie M, Chui L, Svenson LW. Spatiotemporal scan statistics for the detection of outbreaks involving common molecular subtypes: using human cases of Escherichia coli O157:H7 provincial PFGE pattern 8 (National Designation ECXAl.0001) in Alberta as an example. Zoonoses Public Health. 2013;60(5):341-8.

14. Green CG, Krause DO, Wylie JL. Spatial analysis of campylobacter infection in the Canadian province of Manitoba. Int J Health Geogr. 2006;5:2.

15. Wang T, Xue F, Chen Y, Ma Y, Liu Y. The spatial epidemiology of tuberculosis in Linyi City, China, 2005-2010. BMC Public Health.2012; 12:885.

16. Torabi M, Rosychuk RJ. An examination of five spatial disease clustering methodologies for the identification of childhood cancer clusters in Alberta, Canada. Spat Spatiotemporal Epidemiol. 2011;2(4):321-30.

17. Varga C, Pearl DL, McEwen SA, Sargeant JM, Pollari F, Guerin MT. Incidence, distribution, seasonality, and demographic risk factors of Salmonella Enteritidis human infections in Ontario, Canada, 2007-2009. BMC Infect Dis. 2013;13:212

18. Middleton $D$, Savage $R$, Tighe MK, Vrbova L, Walton $R$, Whitfield $Y$, et al. Risk factors for sporadic domestically acquired Salmonella serovar Enteritidis infections: a case-control study in Ontario, Canada. Epidemiol Infect 2013. 2011;142(7):1411-21

19. Nesbitt A, Ravel A, Murray R, McCormick R, Savelli C, Finley R, et al. Integrated surveillance and potential sources of Salmonella Enteritidis in human cases in Canada from 2003 to 2009. Epidemiol Infect. 2012;140(10): 1757-72.

20. Tighe MK, Savage R, Vrbova L, Toolan M, Whitfield Y, Varga C, et al. The epidemiology of travel-related Salmonella Enteritidis in Ontario, Canada, 2010-2011. BMC Public Health. 2012;12(1):310.

21. Arcury TA, Gesler WM, Preisser JS, Sherman J, Spencer J, Perin J. The effects of geography and spatial behavior on health care utilization among the residents of a rural region. Heal Serv Res. 2005;40(1):135-56.

22. Statistics Canada. Table 051-0001. Estimates of population, by age group and sex for July 1, Canada, provinces and territories annual (persons unless otherwise noted). Available at: http://www5.statcan.gc.ca/cansim/ a26? lang=eng\&id $=510001$.

23. Government of Ontario. Health Protection and Promotion Act R.S.O.1990 H. 7. 1990 Part VI. 77.6 (1) - 77.6 (7).

24. Ontario Ministry of Health and Long-Term Care (MOHLTC). Ontario Public Health Standards. Infectious Diseases Protocol. 2015. Available at: http:// www.health.gov.on.ca/en/pro/programs/publichealth/oph_standards/docs/ infectious_diseases.pdf
25. Bale JA, de Pinna EM, Threlfall EJ, Ward LR. Kauffmann-White Scheme 2007: Salmonella Identification - Serotypes and Antigenic Formulae. London: Health Protection Agency; 2007.

26. Ward LR, de Sa JDH, Rowe B. A phage typing scheme for Salmonella enteritidis. Epidemiol Infect. 1987;99:291-304.

27. Statistics Canada. Health region boundary files. Available at: http://www. statcan.gc.ca/pub/82-402-x/2009001/rg-eng.htm

28. Kulldorff M. A spatial scan statistic. Commun Stat. 1997;26:1481-96.

29. Kulldorff M. and Information Management Services, Inc. SaTScanTM v9.0: Software for the spatial and space-time scan statistics. Available at: http:// www.satscan.org

30. Kulldorff M, Athas W, Feuer E, Miller B, Key C. Evaluating cluster alarms: A space-time scan statistic and brain cancer in Los Alamos. Am J Public Health. 1998:88:1377-80.

31. Besag J, Clifford J. Sequential Monte Carlo p-values. Biometrika. 1991;78: $301-30$.

32. World Health Organization. Foodborne disease outbreaks: Guidelines for investigation and control. 2008. Available at: http://www.who.int/foodsafety/ publications/foodborne_disease/outbreak_guidelines.pdf

33. Public Health Agency of Canada. Canada's Foodborne Illness Outbreak Response Protocol (FIORP) 2010: To guide a multi-jurisdictional response. Available at: http://www.phac-aspc.gc.ca/zoono/fiorp-mitioa/index-eng. php\#toc10

34. Kafatos G, Andrews N, Gillespie IA, Charlett A, Adak GK, De Pinna E, et al. Impact of reduced numbers of isolates phage-typed on the detection of Salmonella outbreaks. Epidemiol Infect. 2009;137(6):821-7.

35. Guerin MT, Martin SW, Darlington GA. Temporal clusters of Salmonella serovars in humans in Alberta, 1990-2001. Can J Public Health. 2005; 96(5):390-5.

36. Vrbova L, Johnson K, Whitfield Y, Middleton D. A descriptive study of reportable gastrointestinal illnesses in Ontario, Canada, from 2007 to 2009. BMC Public Health. 2012;12:970.

37. Pires SM, Evers EG, van Pelt W, Ayers T, Scallan E, Angulo FJ, et al. Attributing the human disease burden of foodborne infections to specific sources. Foodborne Pathog Dis. 2009;6:417-24.

38. Kimura AC, Reddy V, Marcus R, Cieslak PR, Mohle-Boetani JC, Kassenborg HD. Chicken consumption is a newly identified risk factor for sporadic Salmonella enterica serotype Enteritidis infections in the United States: a case-control study in FoodNet sites. Clin Infect Dis. 2004;38 Suppl 3:S244-52.

39. Beatty ME, Shevick G, Shupe-Ricksecker K, Bannister E, Tulu A, Lancaster K, et al. Large Salmonella Enteritidis outbreak with prolonged transmission attributed to an infected food handler, Texas, 2002. Epidemiol Infect. 2009; 137(3):417-27.

40. Hedican E, Hooker C, Jenkins T, Medus C, Jawahir S, Leano F, et al. Restaurant Salmonella Enteritidis outbreak associated with an asymptomatic infected food worker. J Food Prot. 2009;72(11):2332-6.

41. Medus C, Smith KE, Bender JB, Leano F, Hedberg CW. Salmonella infections in food workers identified through routine public health surveillance in Minnesota: impact on outbreak recognition. J Food Prot. 2010;73(11):2053-8.

42. Scott E. Food safety and foodborne disease in 21st century homes. Can J Infect Dis. 2003;14(5):277-80.

43. Wagner VR, Silveira JB, Tondo EC. Salmonelloses in the State of Rio Grande do Sul, southern Brazil, 2002 to 2004. Braz J Microbiol. 2014;44(3):723-9.

44. Ethelberg S, Olsen KE, Gerner-Smidt P, Mølbak K. Household outbreaks among culture-confirmed cases of bacterial gastrointestinal disease. Am J Epidemiol. 2004;159(4):406-12.

45. Aiken AM, Lane C, Adak GK. Risk of Salmonella infection with exposure to reptiles in England, 2004-2007. Euro Surveill. 2010;15(22):19581.

46. Sauteur PM, Relly C, Hug M, Wittenbrink MM, Berger C. Risk factors for invasive reptile-associated salmonellosis in children. Vector Borne Zoonotic Dis. 2013;13(6):419-21.

47. Johnson LR, Gould LH, Dunn JR, Berkelman R, Mahon BE, Foodnet Travel Working Group. Salmonella infections associated with international travel: a Foodborne Diseases Active Surveillance Network (FoodNet) study. Foodborne Pathog Dis. 2011;8(9):1031-7.

48. Ravel A, Nesbitt A, Marshall B, Sittler N, Pollari F. Description and burden of travel-related cases caused by enteropathogens reported in a Canadian community. J Travel Med. 2011;18:8-19. 
49. Scallan E, Jones TF, Cronquist A, Thomas S, Frenzen P, Hoefer D, et al. Factors associated with seeking medical care and submitting a stool sample in estimating the burden of foodborne illness. Foodborne Pathog Dis. 2006; 3(4):432-8.

50. MacDougall L, Majowicz S, Doré K, Flint J, Thomas K, Kovacs S, et al. Underreporting of infectious gastrointestinal illness in British Columbia, Canada: who is counted in provincial communicable disease statistics? Epidemiol Infect. 2008;136(2):248-56.

51. Batz MB, Doyle MP, Morris Jr G, Painter J, Singh R, Tauxe RV, et al. Attributing illness to food. Emerg Infect Dis. 2005;11(7):993-9.

52. Jacob CJ, Powell DA. Where does foodborne illness happen-in the home, at foodservice, or elsewhere-and does it matter? Foodborne Pathog Dis. 2009; 6(9):1121-3.

Submit your next manuscript to BioMed Central and we will help you at every step:

- We accept pre-submission inquiries

- Our selector tool helps you to find the most relevant journal

- We provide round the clock customer support

- Convenient online submission

- Thorough peer review

- Inclusion in PubMed and all major indexing services

- Maximum visibility for your research

Submit your manuscript at www.biomedcentral.com/submit 